\title{
Textile EEG Cap using Dry-Comb Electrodes for Emotion Detection of Elderly People
}

\author{
Fangmeng ZENG ${ }^{1}$, Panote Siriaraya ${ }^{2}$, Dongeun $\mathrm{Choi}^{3}$, Noriaki Kuwahara ${ }^{4}$ \\ Graduate School of Science and Technology, Kyoto Institute of Technology, Kyoto, Japan 1,2,3,4 \\ Faculty of Informatics, The University of Fukuchiyama, Kyoto, Japan ${ }^{3}$
}

\begin{abstract}
Emotions are fundamental to human life and can impact elderly healthcare encounters between caregiver and patient. Detecting emotions by monitoring the physical signals with wearable smart devices offers new promises for care support. While there are multiple studies on wearable devices, few of these pertain to soft electroencephalogram (EEG) caps designed for long-time wear by elderly people. In this study, a 4channel textile cap was designed with dry electrodes held by an ultra-soft gel holder, while fashion and ergonomic design features were introduced to enhance wearability and comfort. The dryelectrode textile cap performed highly for monitoring EEG signals; closely matching the wet electrodes equipment. All participants reported positive feedback stating that the textile cap was softer, lighter, and more comfortable than other devices. A cumulative contribution rate of $72.199 \%$ for two factors (materials properties factor and design pattern factor) was achieved using the principal factor method (PFA), which are influencing the usability of the wearable devices. An average emotion classification accuracy of $81.32 \%$ was obtained from 5 healthy elderly subjects. It was thus concluded that the proposed method provides a stable monitoring and comfortable user experience for users, and can be used to detect emotions for elderly people with good results in the future.
\end{abstract}

Keywords-EEG monitoring; elderly people; emotion detection; textile EEG cap; wearing-comfort

\section{INTRODUCTION}

While aging societies are a global phenomenon, with a population share of $23 \%$ in 2009 [1], and an estimated $33 \%$ (one-third) by 2030 [1], Japan has maintained the largest share and fastest-growing population of persons aged 65 years or over in the world. This will bring serious challenges to healthcare and social services. Aging research including mechanisms of aging, detection of disease, and healthcare studies are a major theme in Japanese academic universities and industries. Yet, more research on assessing the quality of life for elderly people is required to strengthen the healthcare system.

Emotions play an important role in people's life, influencing behavior, cognition, and communication. The quality of life for humans could be evaluated according to their emotions. Research on real-time emotion detection using physiological signals has grown rapidly in recent years. The electroencephalogram (EEG) in particular, has been key research focus due to its high accuracy in reflecting emotional states with objective and comprehensive data [2][3]. Studies [4] have shown that emotions are strongly connected to the prefrontal cortex, frontal cortex, and parietal region of the brain. Now, with added advancements in affective computing [5], it's able to recognize emotions from EEG signals using diversified classification methods that extract time and frequency domain features. In the earlier study [6], it's successfully achieved that binary emotional changes were classified using convolutional neural network (CNN) and achieved an EEG signal accuracy of $90 \%$ for two classes on the arousal level. However, the aim in this study is to develop an emotion detecting system using EEG collecting devices that combines accurate signal collection with a comfortable user-friendly experience for elderly people.

The traditional EEG system [7] using wet electrodes with a conductive paste acquires a reliable signal provided there is good contact between the electrode and the scalp through the hair. However, this system [8] depends on the help of professional staff as it takes a long time to clean and prepare the skin. Moreover, long-term skin contact [9] can cause skin irritation, leftover residue on the skin, and drying out of the paste, causing a reduction in signal quality. Overall, wet electrodes are known to cause discomfort and vexation for patients, users and researchers, making them not suitable for extended monitoring, especially for elderly people.

One possible gel-free alternative is the use of dry electrodes to simplify the process. To date, many dryelectrodes EEG devices that take advantage of the low impedance, high signal quality and comfortable user experience [10] offered by dry electrodes have been developed. However, despite the remarkable achievements in EEG devices using dry electrodes, such as DSI 10/20 by Quasar, MindWave Mobile 2 by NeuroSky, EPOC+ by Emotiv, the frames of these devices are often made from plastic [11] which is bulky, hard, and mechanical; deterring users from using it [12]. On the other hand, textile frames are skin-friendly, soft to touch, comfortable to wear and visually appealing. While many textile caps — such as MCScap by Medical Computer Systems, and eego by Ant Neuro-which accurately and consistently monitor the energy signal have been developed, most of these designs are not user-friendly. They wrap tightly around the head through the chin and ears, to ensure the stable contact between the electrode and the scalp. For long-term use, it causes a sense of restraint and an uncomfortable pressure. Therefore [13][14], it is necessary to develop a wearable device for elderly people, which not only obtains a reliable signal, but is also user-friendly, has an easy light-weight mounting and un-mounting headset, is ergonomically designed for extended wear, and is visually 
appealing. At present, there are limited studies that focus on these factors.

This paper explains the research methods and processes for successful development of a comfortable and stylish EEG cap using dry electrodes for daily monitoring of elderly people. The performance of the cap is discussed and the emotion classification on elderly people is also presented. This paper is organized as follows: Section II explained the design of the textile cap in detail; In Section III, the experiments on performance evaluation and emotion detection were presented; Section IV described the results and discussion of: the signal quality compared with traditional wet electrodes; the usability performance compared with other wearable device; the implementation of emotion detection; Section $\mathrm{V}$ drew conclusions and Section VI summarized the limitations and provided suggestions in future work.

\section{DESIGN OF THE TEXTILE EEG CAP}

An overview of the development was presented in this section. First, the number and position of the electrodes for the brain cap prototype was determined. A blueprint for a $3 \mathrm{D}$ printed mold was then designed and printed. Subsequently, a super-soft electrode holder was made using urethane resin, and a 4-electrode textile brain cap was designed with an aesthetic focus.

\section{A. The Gel-based Holder Design}

The distribution and number of electrodes were determined based on emotion detection and wearing experience. The F3, F4, P3, and P4 points distributed in the frontal and parietal cortex were selected as EEG channels in accordance with the International 10-20 system.

Reusable dry comb electrodes provided by the OpenBCI platform were adopted along with 5mm-long blunt prongs used to accommodate longer hair and enhance scalp contact. An ultra-soft gel-based electrode holder was introduced to maintain stable contact between the dry electrodes and the scalp without strong pressure. A 3D printing mold was needed to construct the ultra-soft gel-based electrode holder. The production details of the 3D printing mold are as follows.

1) $3 \mathrm{D}$ printing mold design: The average head circumference of the human adults [15] was estimated at about $55 \mathrm{~cm}$ in females and $57 \mathrm{~cm}$ in males. A medium-sized prototype based on the average head circumference was planned. First, we combined the International 10-20 system measurement [16] to determine the size of the EEG electrode holder mold. The distance between F3 and F4, P3 and P4, was set as $100 \mathrm{~mm}$. The total length of the mold inside was $150 \mathrm{~mm}$, the width inside was $50 \mathrm{~mm}$ and the height was $8 \mathrm{~mm}$. The size of the two columns was devised based on the electrode parts. As the human-skin gel has high elasticity and softness, the bottom diameter was set as $10 \mathrm{~mm}$ so that the electrode with the protector could cross smoothly. The top diameter was set to $5 \mathrm{~mm}$ to hold the protector firmly. In order to easily remove the gel, the column height which needed to be higher than $8 \mathrm{~mm}$ was settled at $10 \mathrm{~mm}$. Fig. 1 shows the design of a $3 \mathrm{D}$ printing mold for the EEG electrode holder.

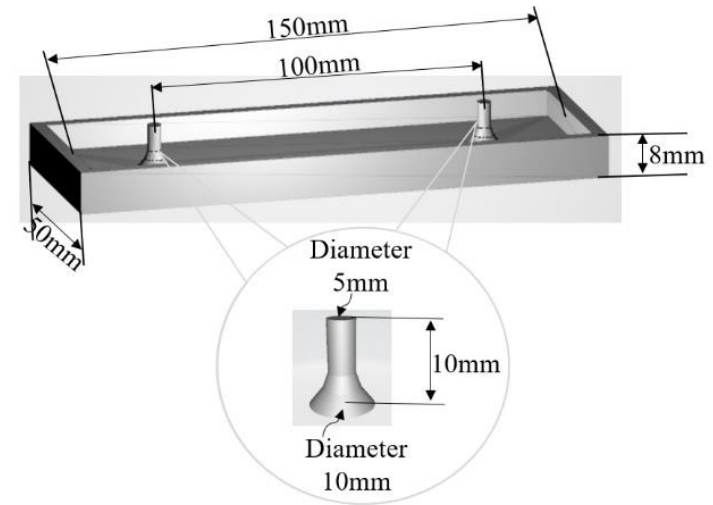

Fig 1. The Design of the 3D Printing Mold of the Electrode Holder.

3D printing was done at university. Once the final design was achieved, the parts were printed using a Polylactic Acid (PLA) plastic by Value 3D MagiX MF-1000.

2) Development of the ultra-soft gel holder: The ultra-soft gel made by urethane resin was produced by mixing two liquids (the base and hardener by a weight ratio of $3: 1$ ). The volume of the mold was calculated before making the gel and the mold was sprayed with special slide urethane mold released 1-hour in advance. The production steps were carried out following the Monotaro platform instructions. Fig. 2 shows the schematic diagram for producing the electrode gel holder. The gel product exhibits the appropriate level of elasticity and ultra-softness. As for a 4-channel cap, one-gel product holding 2 electrodes, 2 gel holders were made.

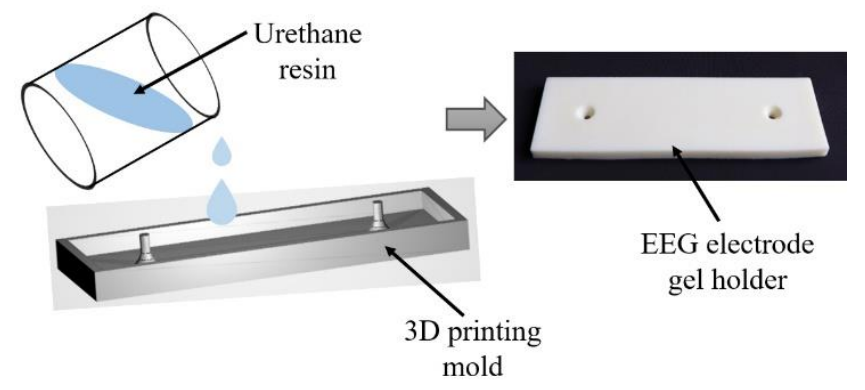

Fig 2. The schematic diagram for producing the gel holder.

\section{B. Design of the Textile EEG Cap}

With considerations toward comfort, flexibility, and appearance, a soft textile cap for EEG was put forward as the main candidate in this research.

An elastic fabric made of rayon (R) 43\%, polyester (P) $51 \%$, polyurethane (PU) $6 \%$, was used as a ground cloth to make a fitted cap based on the average medium head size. After that, the ultra-soft gel was covered with fabric, two holes were made on the fabric to set the electrodes, and the fabric adhered to the cap. The electrodes were crossed through the holes in the gel holder and the fabric was then connected to the blue protector with screws. 4 electrodes held separately with 2 gel holders, created as per above, were then fixed to the textile cap. The softness and flexibility enabled the gel holder 
to adjust its shape to fit the head in the same manner as textiles. The electrodes were then placed on the scalp and stabilized with the holder. Fig. 3 illustrates the design and structure of the textile EEG cap equipped with dry electrodes.

\section{EXPERIMENTS AND METHODS}

This part presents the experiments to evaluate the signal performance of the designed textile EEG cap and to detect emotions for elderly people.

\section{A. Experiment I - Textile EEG Cap Testing}

A pilot experiment was conducted on healthy young people to evaluate the signal measuring and usability of the textile EEG cap. 20 students (13 females and 7 males) took part in the experiment. Before the experiment, each subject was asked to measure their head circumference. The head sizes ranged from $(56 \sim 58) \pm 0.5 \mathrm{~cm}$. The textile EEG cap was then fitted using the adjustment features. All participants consented to the content of the experiment.

After the textile cap was set up on their head and the stability of the signal was checked, the subject was asked to relax for a moment, and then close their eyes for 4 minutes and listen to a song. Next, they were asked to open their eyes for 4 minutes and look at the lyrics while listening to a song. The EEG signals from frontal and parietal areas (F3, P3, F4 and P4) were collected by the textile cap. The reference and ground electrodes were placed on the ears using ear-clips. The signals were recorded with a Cyton board and shown on the OpenBCI GUI in real-time. For comparison, each subject was asked to complete the experiment with both the gold cup wet electrodes (using Ten20 conductive paste), the OpenBCI Ultracortex
Mark $\chi$ headset, and the soft EEG textile cap in the same manner. The three EEG devices were set up in random. The medium Mark IV frame was 3D printed and self-assembled following the instructions mentioned on the OpenBCI website. The set-up time for each device was then recorded. At 30 minutes and more, the wet electrodes set-up was the most time-consuming. The set-up of the textile cap and Mark IV, which was recorded at approximately $12 \mathrm{~min}$ and $15 \mathrm{~min}$ respectively, took the least amount of time. From the experiment, it was clear that the wearable devices equipped with dry electrodes were more efficient and convenient.

At the end of the experiment, each subject was asked to scale the two wearable devices (Ultracortex Mark IV and textile EEG cap), using a semantic differential (SD) evaluation survey. Each subject was given an explanation of the question in advance and allowed to physically touch and observe the two devices. Semantic differential (SD) is a type of rating scale designed by Osgood et al. [17], to measure the attitude towards the given object, event or concept. In this study for wearable devices, user-friendly characteristics such as simple operation, no mechanical stress during longer-term use, and daily appearance were taken into account. In the experiment, seven contrasting adjectives were used based on these matters, and a 7-point rating scale from -3 to +3 to derive the attitudes of the participants towards the two wearable devices. The seven bipolar adjectives were: 1.tight/loose, 2.strong/weak, 3.heavy/light, 4.rigid/soft, 5.bad-looking/good-looking, 6.uncomfortable/comfortable, 7.dislike/like. For each antonym adjective pair, there was an explanatory question (see Appendix).

The total experiment process for each subject was explained in Fig. 4, taking 2 hours. Trial 1, trial 2 and trial 3 was conducted randomly, with a $3 \mathrm{~min}$ break after each trial.

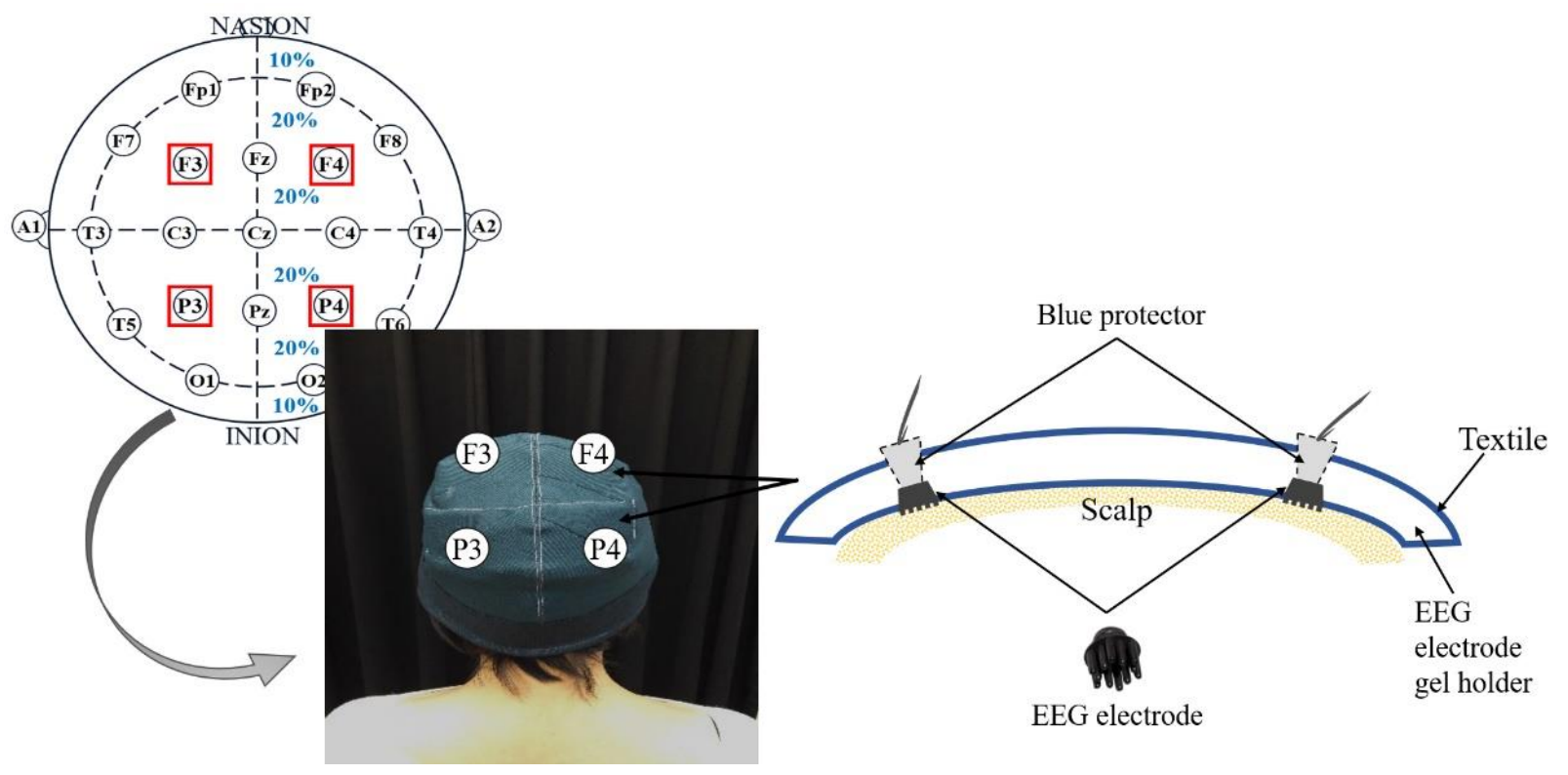

Fig 3. The Design and Structure of Textile EEG Cap with Dry Electrodes held by Ultra-Soft Gel Holders. 


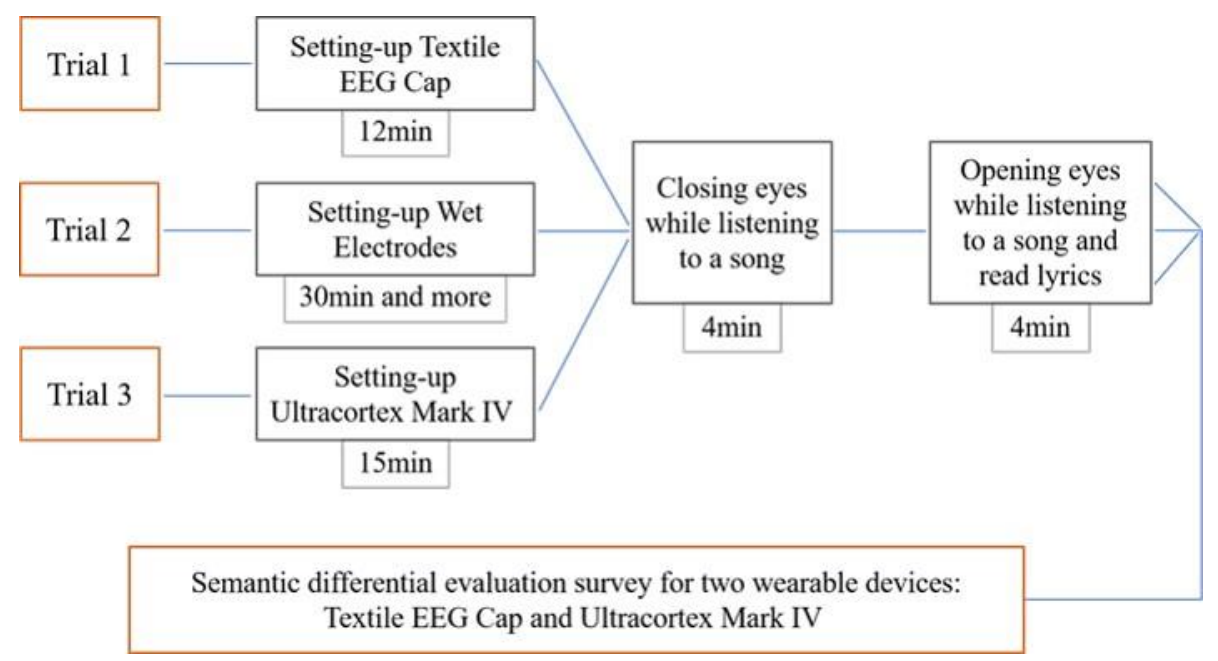

Fig 4. The Total Process of the Experiment.

\section{B. Experiment II - Emotion Detection for Elderly People}

To further test the application performance of the textile EEG cap, caps in two sizes were made: $\mathrm{S}$ size for head circumference ranging from $(55 \sim 57) \pm 0.5 \mathrm{~cm}$, L size for head circumference ranging from (57 60) $\pm 0.5 \mathrm{~cm}$. An emotion monitoring experiment was conducted on 5 healthy elderly subjects ( 2 females and 3 males, aged over 65 years old). A detailed file containing general information and instructions on the experiment was sent to them in advance. In this file, they were required to wear loose clothes and women were asked to wear their hair naturally to help facilitate the setup of the devices. All the subjects signed a consent form stating that their physical information could be used for this research and not for other use. A Japanese student was available for any questions from Japanese elderly participants.

In this experiment, videos were chosen to elicit positive and negative emotions. Six 1-3 min film-clips [18] were selected based on previous studies. Of these, 3 videos elicited positive emotion and 3 videos for negative emotion. Japanese professors and teachers confirmed the final video selection, as elderly people may be vulnerable to mental and physical stress or affected in daily life by the videos.

The experiment process was first explained to the elderly subjects clearly in Japanese in advance. Then the head size of each subject was measured to set with the correct size of cap. Next, the textile EEG cap was equipped to the subjects with four channels (F3, F4, P3 and P4) and two ground channels clipped to their ears. At the same time, the self-assessment form was explained in Japanese so that the subject got familiarized with the content. Before the formal experiment, the subjects were asked to sit comfortably with minimal movement during the experiment, and the signal stability was checked. After that, the subjects performed a practice trial to familiarize themselves with the system. Next, the formal experiment started with $1 \mathrm{~min}$ of baseline recording while the subjects were asked to close their eyes. The six videos were presented semi-randomly in six trials, taking about $10 \mathrm{~min}$ in total. Each trial consisted of the following steps: (1) 10s to relax and display the current number of the displayed videos; (2) Display of videos; (3) 20s of self-assessment.

EEG signals were collected on an equipped recording PC (Dell Inspiron 7370), sampling at 250Hz. Stimulating videos were performed on a PC (Dell Alienware M15). Fig. 5 shows the scene of one subject during the experiment. At the end of the experiment, the subjects were asked to evaluate the wearing experience of the textile EEG cap.

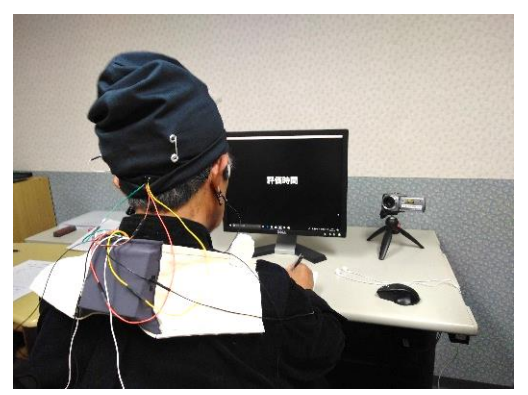

Fig 5. A Subject during the Emotional Detection Experiment.

\section{RESULTS AND DISCUSSION}

\section{A. The Performance of Textile EEG Cap on Signal Capture}

The measured data of 2 subjects was incomplete as the wet electrodes lost contact with the scalp. There was some obvious noise present in the data from another 2 subjects due to movement and the heaviness of hair. Therefore, the signal data of 16 participants were used for data analysis. The raw data was filtered with a bandpass $(0.5 \sim 40 \mathrm{~Hz})$ to remove the main noise and artifacts in order to get clean data. Fig. 6 showed the filtered signal waveform of the P3 channel collected from the textile EEG cap.

During the frequency analysis, the power spectral density (PSD) was obtained using a multi-taper spectral estimation method [19], which is more robust than the classical and Welch's periodograms. At last, the ratio of each band power (delta: $0.5 \sim 4 \mathrm{~Hz}$, theta: $4 \sim 8 \mathrm{~Hz}$, alpha: $8 \sim 12 \mathrm{~Hz}$, beta: $12 \sim 40 \mathrm{~Hz}$ ) in 10 s was computed through integration. 


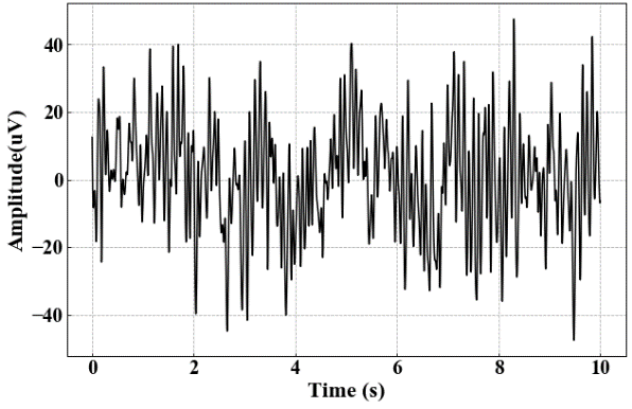

Fig 6. The Filtered Waveform of P3 Measured from Textile EEG Cap.

Table I lists the average ratios of the band power of the EEG signal of each channel collected from the textile cap for the 16 subjects, with both eyes closed and eyes opened. It was found that the alpha band power was higher in each channel with eyes closed. The alpha wave was more apparent in the parietal lobe (P3 and P4) than the frontal lobe (F3 and F4). In general, the delta wave tends to be seen commonly in adults in slow-wave sleep state, while theta wave may be seen in a drowsy or meditative state. The alpha wave emerges with the closing of the eyes or when in a state of relaxation and decreases with the opening of the eye or mental exertion. Beta wave is closely linked to active thinking and attention. It was deduced that the textile EEG cap could detect EEG signals from all the channels correctly and stably reflect the brain activities.

For comparison of the signal quality, the EEG signals collected from the textile cap were compared with the signals collected with wet electrodes. As the signals were not monitored simultaneously, the PSD of the signals from each channel between the textile cap and wet electrodes was compared. The correlation coefficient (CC) was widely used to measure the degree of linear correlation between two sets of data. Fig. 7 shows a comparison of one sample of PSD of the signals collected using the textile EEG cap system with the wet electrodes system with (a) eyes closed and (b) eyes opened respectively. Table II presents the average correlation results of the PSD of signals from all channels from these two types of systems. The results in Table II showed that all the correlation values are over 0.820 , with an average of about 0.857 for closed eyes and opened eyes. Hence, the measured signals from the textile cap and the wet electrodes were highly correlated to each other.

The above results show that the designed textile EEG cap monitored EEG signals from all channels and reflected brain activity to the same level of accuracy as the wet electrodes. This was due to the reliable contact between the new dry electrodes and the scalp which was supported by the ultra-soft gel holder. As shown in Fig. 7, when the eyes were opened some noises, such as eye blinking, were easily picked up in the low-frequency band. This highlighted the need to improve the noise-immunity of the dry electrodes and the structure of the device. On the other hand, it also revealed that a strong denoising algorithm can be achieved.

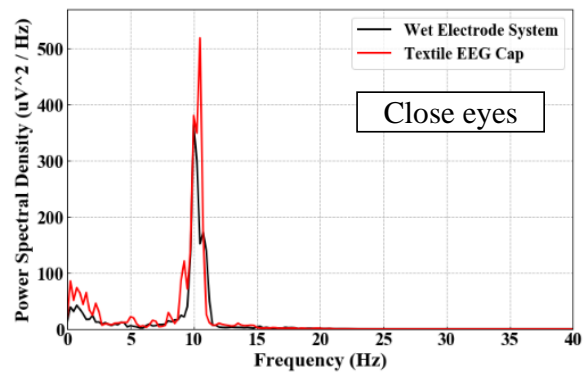

(a)

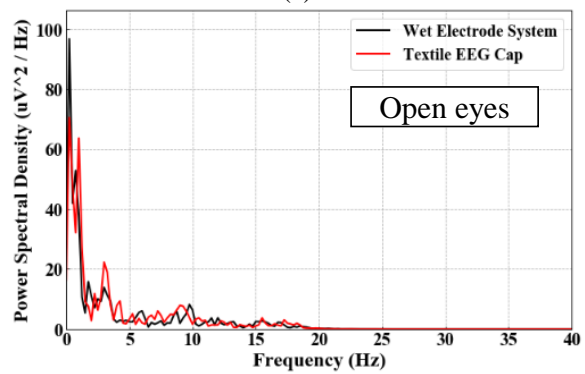

(b)

Fig 7. The Comparison of PSD of EEG Signal recorded by Textile EEG Cap and Wet Electrodes System respectively (a) When Close Eyes, (b) When Open Eyes.

TABLE I. THE AVERAGE RATIOS OF BAND POWER OF EEG SIGNALS FROM EACH CHANNEL COLLECTED FROM THE TEXTILE EEG CAP WHEN CLOSE EYES AND OPEN EYES

\begin{tabular}{|l|l|l|l|l|l|l|l|}
\hline \multicolumn{2}{|l|}{ Close eyes } \\
Channel
\end{tabular}


TABLE II. THE AVERAGE CORRELATION COEFFICIENTS OF THE PSD OF EEG SIGNAL OF EACH CHANNEL WITH EYES CLOSED AND EYES OPENED, BETWEEN THE TEXTILE EEG CAP AND WET ELECTRODES SYSTEM

\begin{tabular}{|l|l|l|}
\hline \multicolumn{1}{|c|}{ State } & Close eyes & Open eyes \\
\hline F3 & 0.829 & 0.835 \\
\hline P3 & 0.823 & 0.896 \\
\hline F4 & 0.896 & 0.900 \\
\hline P4 & 0.849 & 0.832 \\
\hline
\end{tabular}

\section{B. Factor Analysis of Wearable Devices}

Fig. 8 visualizes the image scale of the semantic differential (SD) evaluation average score of the 20 subjects for the two wearable devices: the textile EEG cap and the Ultracortex Mark IV. The soft textile EEG cap attracted more positive feedback than Ultracortex Mark IV. The textile cap was also reported to exert less pressure and skin irritation than the Mark IV. Moreover, the majority of the subjects expressed that they felt the textile cap was very light-weight, soft, more natural in appearance, and able to be worn as an accessory in daily life. Most people also exhibited a preference for the textile cap for long-term use due to the added comfort and natural appearance.

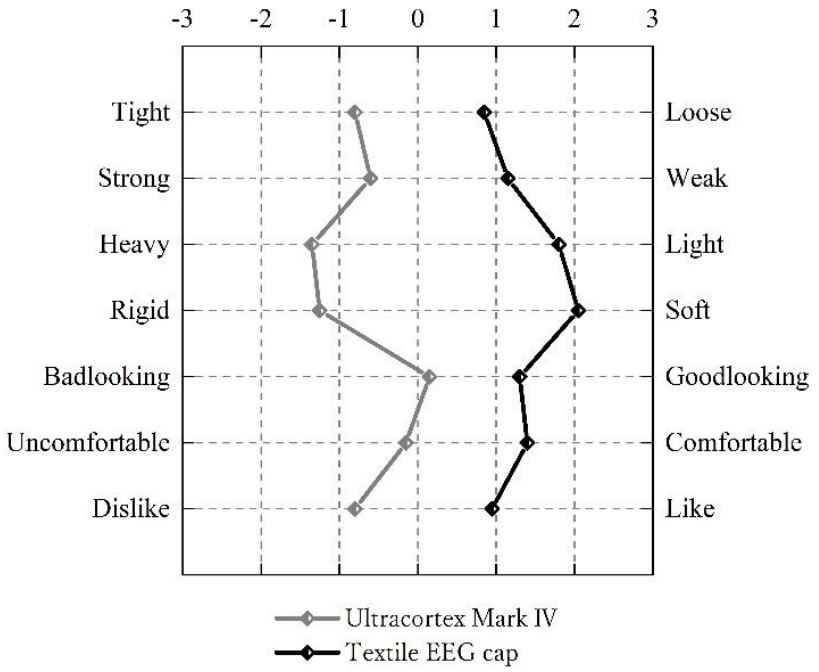

Fig 8. Image Scale of SD Evaluation Score (Average Value) of the Two Wearable Devices.

The positive feedback from subjects for the EEG textile cap may be attributed to the following factors. Firstly, the frame of the cap was sewn with elastic textile fabric made of $43 \%$ rayon, $51 \%$ polyester, $6 \%$ polyurethane. The combination of rayon (R) for comfort, polyester (P) for strength and polyurethane (PU) for flexibility optimized the user experience. Secondly, the ultra-softness and sound elasticity of ultra-soft gel exerted a certain buffering effect on the scalp to relieve the pressure around the head. The gel acted like human skin. Thirdly, the use of the gel allowed for a greater focus on the visual and ergonomic components of a fitted cap without tightly surrounding the chin or ears. The users reported enjoying the good fit and freedom it provided. The Ultracortex Mark IV design utilizes a large rigid plastic frame for mounting the electrodes, which are fixed using extra plastic inserts. Despite the adjustable 3D-printable EEG headset that receives signals while reducing noise, its added weight, rigidness, and appearance limited its application for extended use in daily life.

A factor analysis of SD scores of the two wearable devices (the textile EEG cap and the Ultracortex Mark IV) taken from the 20 subjects was conducted. The purpose was to gain insight into the underlying factors which influence the usability of wearable EEG devices. Factor analysis [20] is a statistical technique that extracts fewer common unobserved factors from observed and correlated variables.

Python was used to perform the factor analysis. Firstly, the Kaiser-Meyer-Olkin (KMO) and the Bartlett test was conducted to check the suitability of data for factor analysis. In general, the value of KMO is less than 0.6 and is considered inadequate [20]. Table III presents the results from the KMO and the Bartlett test. It shows that the overall KMO value for the data is $0.83(>0.6)$, which indicates that it could achieve a sound analysis effect. The p-value in the Bartlett test is 0.00; indicating that the correlation matrix is not an identity matrix. Therefore, the factor analysis was proceeded.

TABLE III. THE KMO AND BARLETT'S TEST OF THE FACTOR ANALYSIS OF THE WEARABLE DEVICES

\begin{tabular}{|c|l|}
\hline Kaiser-Meyer-Olkin Measure of Sampling Adequacy. & 0.830 \\
\hline Bartlett's Test of Sphericity Approx. Chi-Square & 222.205 \\
\hline Sig. & 0.000 \\
\hline
\end{tabular}

Secondly, after selecting the number of factors, a scree plot was used to draw a straight line for each factor and its eigenvalues. The position where the number of eigenvalues is greater than 1.0 is used to help determine the appropriate number of factors. Fig. 9 shows the scree plot of the factor analysis for the two wearable devices. It can be seen that only 1 factor has an eigenvalue greater than 1.0. However, as the second-factor eigenvalue is very close to 1.0 , it was difficult to select the number of factors. As a result, combing interpretation of variation ratio of principle component analysis was adopted.

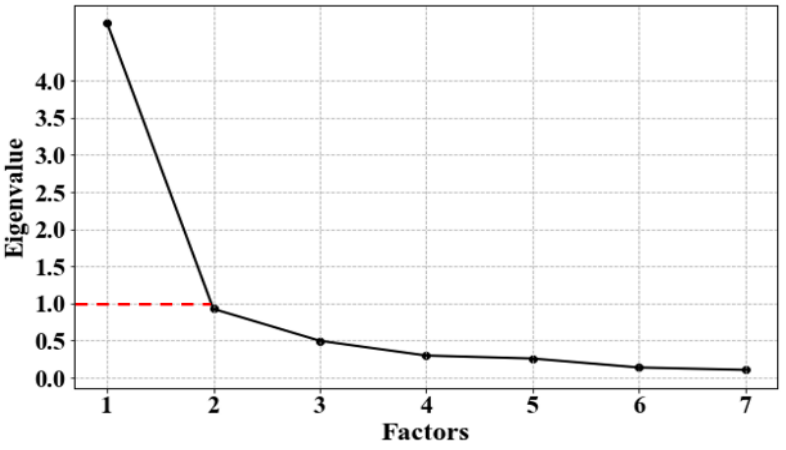

Fig 9. The Screen Plot of Factor Analysis of the Wearable Devices. 
TABLE IV. TOTAL VARIANCE EXPLAINED OF EACH COMPONENT OF THE FACTOR ANALYSIS OF THE WEARABLE DEVICES

\begin{tabular}{|c|c|c|c|c|c|c|}
\hline \multirow[b]{2}{*}{ Component } & \multicolumn{3}{|c|}{ Initial Eigenvalues } & \multicolumn{3}{|c|}{ Rotation Sums of Square Loadings } \\
\hline & Total & of Variance \% & Cumulative \% & Total & of Variance \% & Cumulative \% \\
\hline 1 & 4.780 & 68.279 & 68.279 & 3.337 & 47.675 & 47.675 \\
\hline 2 & 0.926 & 13.233 & 81.513 & 1.717 & 24.524 & 72.199 \\
\hline 3 & 0.497 & 7.096 & 88.609 & & & \\
\hline 4 & 0.298 & 4.256 & 92.865 & & & \\
\hline 5 & 0.258 & 3.684 & 96.549 & & & \\
\hline 6 & 0.137 & 1.957 & 98.507 & & & \\
\hline 7 & 0.105 & 1.493 & 100.000 & & & \\
\hline
\end{tabular}

It has been suggested that the extracted principal component should explain at least 5-10\% [20] of the data variation. It was also believed that the extracted principal components should accumulate $60-70 \%$ of the data variation cumulatively. The principal factor method with varimax (orthogonal rotation) was used to get the total variance explained. Table IV describes the result of the total variance explained in each component. These results showed that the second component explained $13.23 \%$ of the data variation and the first two components cumulatively explained $72.199 \%$ of the data variation. Therefore, the number of factors were fixed at two to perform the required extraction of factors. The rotated component matrix, showing the variance explained by the observed variables, was calculated using the principal factor method with varimax rotation.

Table V shows the rotated component matrix. It shows that the seven variables could be reduced into two factors. Factor 1; the material properties factor, includes 4 variables: 1 . light pressure, 2. weak skin irritation, 3. light-weight, and 4. soft. Factor 2; the design pattern factor, contains 3 variables: 5. good-looking, 6. comfortable, and 7. like. It was shown that the extraction of two factors has a good ability to interpret the results; it is consistent with the SD scale results; and that the textile cap received more positive feedback than the plastic cap. In addition, the variable 6 (comfortable) and variable 7 (like) load in factor 1 was more than 0.5. This shows that factor 1 (materials properties) also impacts them. This factor was expected to play an important role in developing wearable EEG devices in the future.

\section{Emotion Detection Result}

As explained in the section IV, sub-section A, the collected data was filtered to first get clean data first and then extract features. To enable emotion classification, the last 30 seconds of the six videos were used according to the assessment of the subjects. In referring to the algorithm [6], the spectrograms of EEG data were obtained using the Shorttime Fourier transform (STFT), then emotion classification on valence level (positive and negative) using the convolutional neural network $(\mathrm{CNN})$ was conducted.
TABLE V. THE ROTATED COMPONENT MATRIX OF THE FACTOR ANALYSIS OF THE WEARABLE DEVICES

\begin{tabular}{|l|l|l|}
\hline & Factor1 & Factor2 \\
\hline 1 & 0.796 & 0.339 \\
\hline 2 & 0.779 & 0.376 \\
\hline 3 & 0.892 & 0.143 \\
\hline 4 & 0.768 & 0.361 \\
\hline 5 & 0.127 & 0.680 \\
\hline 6 & 0.603 & 0.696 \\
\hline 7 & 0.589 & 0.591 \\
\hline
\end{tabular}

As emotion responses last for just a few milliseconds [21], one spectrogram represents a signal of one-second. In [22], the relationship and differences between the left and right hemispheres are closely related to positive and negative emotions. Each person watching 6 videos, totaling 180s, meant that 720 spectrograms from four channels (F3, F4, P3 and P4) were considered as input. The CNN model had three convolutional layers and three max-pooling layers. The size of all the filters in convolutional layers was $3 \times 3$. A dropout of 0.3 was used after the fully connected layer with 256 hidden layers. The Rectified Linear Unit (ReLU) activating function was used and the applied optimizer was stochastic gradient descent (SGD), with a learning rate of 0.0001. For each subject, classification was performed for the signals from four channels and a 5-fold cross-validation was used to determine the accuracy. Some subjects had a poor accuracy rating of under $70 \%$. According to the videos which were recorded of the experimental scenes, the elderly subjects tend to take naps while watching the stimuli. Also, they had misclassifications during assessment, rating a negative video as positive. The misclassified data was changed to match the correct videos before classifying the emotion. In this way, the results, as shown in Fig. 10 rose to an average accuracy of $81.32 \%$. 


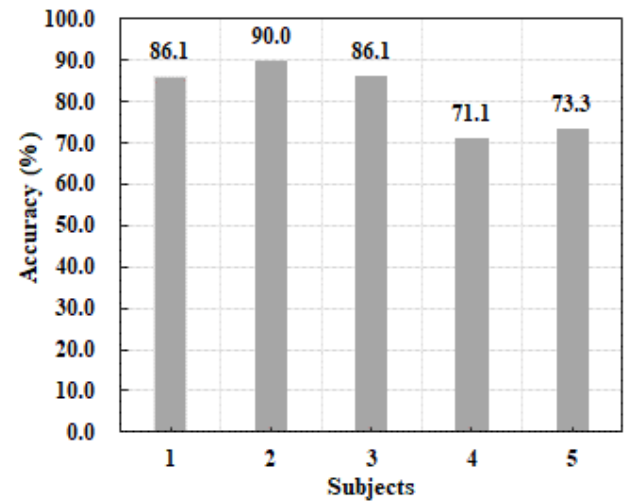

Fig 10. Emotion Classification Accuracy of Elderly People using the Textile EEG Cap.

This result drew out the following points. Firstly, although the classification result on the valence level is worse than the arousal level using the same algorithm, the textile EEG cap was useful in detecting emotions by monitoring EEG data. It's easy to detect whether the emotion was activated or not, however it's hard to distinguish the different emotions. Secondly, to do the experiment with elderly people, more attention should be paid to their physical condition and psychological stress. Thirdly, EEG metrics, such as alpha, correlated with ages, differing between young people and elderly people [23]. The classification algorithm for elderly people needs to be improved. On the other hand, all the subjects considered that the textile cap was comfortable during monitoring, was visually appealing, and could be used in daily life.

Referring to [24], a wearable EEG headband using printed electrodes was developed aiming at supporting elderly people. In consistent with this research, emotion detection using wearable smart devices shows great value to evaluating the quality of life of people. Unlike designing headband tested on young participants, a textile cap was developed with aesthetics and comfort in this research, also measured on elderly people. On the other hand, they designed the energy harvester taking the place of battery, which is the issue to be considered.

\section{CONCLUSIONS}

This paper has presented the development of a comfortable and user-friendly 4-channel EEG cap integrated with textiles which can be used for emotion detection for elderly people. First, the dry comb electrodes were used and secured in place using an ultra-soft urethane gel holder. Next, an ergonomically designed fitted cap was fabricated with a focus on aesthetics and comfort. The wearable device demonstrated high-level performance, reflecting brain activity from all channels. The signals from the cap were strongly paralleled with the commercial wet electrodes, giving an average correlation results of the PSD of EEG signal from all channels about 0.857 . The flexible design improved the usability of the device, enhanced operational conveniences, reduced pressure, and made the device lighter, softer, more comfortable, and more natural in appearance. According to the factor analysis on the SD scale results of the two wearable devices: Textile EEG Cap and Ultracortex Mark IV, the two extracted factors; the materials properties factor and the design pattern factor cumulatively explained $72.199 \%$ of the data variation. This highlights the need to focus on the two factors when designing new wearable headsets in the future. Finally, an emotion detection experiment was performed on five healthy elderly participants to evaluate the application performance of the textile cap, which got an average classification accuracy of $81.32 \%$. The pilot study confirmed the feasibility and prospect of the wearable textile cap in real-time emotion classification for elderly people comfortably.

\section{FUTURE WORK}

This research provides an innovative approach to the development of a comfortable wearable EEG devices. However, there also remains several limitations. Firstly, the cap only has four channels, which needs to include more channels for more EEG information; Secondly, the dry electrodes are commercially available, and would be developed using smart textiles in future work. Thirdly, the power supply comes from the heavy battery, limiting the mobility. Besides, with further research, this would also meet demands to optimize the appearance design, and combine function, ergonomics, and security issues to eliminate reluctances to use the devices from the users, especially elderly people. In the future, it's necessary to contribute to improving the classification algorithm for emotion detection of elderly people. It's promising to realize real-time emotion monitoring system from EEG signal using wearable device during long-term period comfortably and conveniently. Besides EEG cap, wearable devices would be designed to monitor other physical signals, like electrocardiogram (ECG), combined with EEG to detect emotion for elderly people more accurately in daily life.

\section{ACKNOWLEDGMENT}

This research was partially supported by the JSPS KAKENHI Grant Number $19 \mathrm{H} 01138$ and 19H04154. We would also like to extend our gratitude and acknowledgment to the China Scholarship Council (CSC) for supporting the Ph.D. research of F.M.Z., and to all the young and the elderly subjects from Kyoto who cooperated to aid in completing the experiment.

\section{REFERENCES}

[1] N. Muramatsu, and H. Akiyama, "Japan: super-aging society preparing for the future," The Gerontologist, vol. 51, pp. 425-432, 2011.

[2] H. Chao, L. Dong, Y. Liu, and B. Lu, "Emotion Recognition from Multiband EEG Signals Using CapsNet," Sensors, vol. 19, pp. 2212, May 2019.

[3] J. Zhang, M. Chen, S. Zhao, S. Hu, Z. Shi, and Y. Cao, "ReliefF-based EEG sensor selection methods for emotion recognition," vol. 16, pp. 1558, 2016.

[4] J. Kissler, C. Herbert, I. Winkler, and M. Junghofer, "Emotion and attention in visual word processing-An ERP study," Biological Psychology, vol. 80, pp. 75-83, 2009.

[5] D. W. Chen, R. Miao, W. Q. Yang, Y. Liang, H. H. Chen, L. Huang, et al, "A Feature Extraction Method Based on Differential Entropy and Linear Discriminant Analysis for Emotion Recognition," Sensors, vol. 19, pp. 1631, 2019.

[6] F. M. ZENG, P. J. LIAO, M. Iwamoto, and N. Kuwahara, "Emotional Changes Detection for Dementia People with Spectrograms from Physiological Signals," IJACSA, vol. 9, 2018. 
[7] J. W. Kam, S. Griffin, A. Shen, S. Patel, H. Hinrichs, H. Heinze, et al, "Systematic comparison between a wireless EEG system with dry electrodes and a wired EEG system with wet electrodes," NeuroImage, vol. 184, pp. 119-129, 2019.

[8] P. Fiedler, P. Pedrosa, S. Griebel, C. Fonseca, F. Vaz, E. Supriyanto, et al, "Novel multipin electrode cap system for dry electroencephalography," Brain Topography, vol. 28, pp. 647-656, 2015.

[9] G. D. Flumeri, P. Arico, G. Borghini, N. Sciaraffa, A. D. Florio, and F. Babiloni, "The dry revolution: Evaluation of three different EEG dry electrode types in terms of signal spectral features, mental states classification and usability," Sensors, vol. 19, pp. 1365, 2019.

[10] X. Xing, Y. Wang, W. Pei, X. Guo, Z. Liu, F. Wang, et al, "A highspeed SSVEP-based BCI using dry EEG electrodes," Scientific Reports, vol. 8, 2018.

[11] W. D. Hairston, K. W. Whitaker, A. J. Ries, J. M. Vettel, J. C. Bradford, S. E. Kerick, et al, "Usability of four commercially-oriented EEG systems," Journal of Neural Engineering, vol. 11, 2014.

[12] S. Debener, R. Emkes, M. De Vos, and M. G. Bleichner, "Unobtrusive ambulatory EEG using a smartphone and flexible printed electrodes around the ear," Scientific Reports, vol. 5, 2015.

[13] D. L. Lacko, J. Vleugels, E. Fransen, T. Huysmans, G. De Bruyne, M. M. Van Hulle, et al, "Ergonomic design of an EEG headset using 3D anthropometry," Applied Ergonomics, vol. 58, pp. 128-136, 2017.

[14] X. Yu, and W. Qi, "A User Study of Wearable EEG Headset Products for Emotion Analysis," Proceedings of the 2018 International Conference on Algorithms, Computing and Artificial Intelligence. ACM, 2018.

[15] R. P. Ching, "Relationship between head mass and circumference in human adults University of Washington," Technical Brief, 2007.

[16] V. Jurcak, D. Tsuzuki, and I. Dan, "10/20, 10/10, and 10/5 systems revisited: their validity as relative head-surface-based positioning systems," Neuroimage, vol. 34, pp. 1600-1611, 2007.

[17] C. E. Osgood, J. S. George, H. T. Percy, "The measurement of meaning," University of Illinois Press, 1957.

[18] T. L. Gilman, R. Shaheen, K. M. Nylocks, D. Halachoff, J. Chapman, J. J. Flynn, et al, "A film set for the elicitation of emotion in research: A comprehensive catalog derived from four decades of investigation," Behavior research methods, vol. 49, pp. 2061-2082, 2017.

[19] M. J. Prerau, R. E. Brown, M. T. Bianchi, J. M. Ellenbogen, and P. L. Purdon, "Sleep neurophysiological dynamics through the lens of multitaper spectral analysis," Physiology, vol. 32, pp. 60-92, 2016.

[20] A. G. Yong, and P. Sean, "A beginner's guide to factor analysis: Focusing on exploratory factor analysis," Tutorials in Quantitative Methods for Psychology, vol. 9, pp. 79-94, 2013.

[21] M. Codispoti, M. Mazzetti, and M. M. Bradley, "Unmasking emotion: Exposure duration and emotional engagement," Psychophysiology, vol. 46, pp. 731-738, 2009.

[22] J. W. Matiko, S. P. Beeby, and J. Tudor, "Fuzzy logic based emotion classification," 2014 IEEE International Conference on Acoustics, Speech and Signal Processing (ICASSP) IEEE, 2014.

[23] J. P. Trammell, P. G. MacRae, G. Davis, D. Bergstedt, A. E. Anderson, "The Relationship of Cognitive Performance and the Theta-Alpha Power Ratio Is Age-Dependent: An EEG Study of Short Term Memory and Reasoning during Task and Resting-State in Healthy Young and Old Adults," Frontiers in Aging Neuroscience, vol. 9, pp. 364, 2017.

[24] J. W. Matiko, Y. Wei, R. Torah, N. J. Grabham, G. Paul, S. P. Beeby, et al, "Wearable EEG headband using printed electrodes and powered by energy harvesting for emotion monitoring in ambient assisted living," Smart Materials and Structures, vol. 24, 2015.

\section{APPENDIX}

The explanatory questions for the contrasting adjectives.

1) What are your thoughts on the pressure caused by the device?

2) What do you think about the skin irritation caused by the device?

3) Do you think it's heavy?

4) How does it feel to touch?

5) What do you think about its appearance design?

6) How about your feelings on the cap overall?

7) Do you prefer this device for long-term use? 